\title{
水溶液中 2,4,6-三溴苯酚在银阴极上的选择性脱卤
}

\author{
朱英红徐颖华 马昊赵峰鸣 马淳安*
}

(浙江工业大学化学工程与材料学院, 绿色化学合成技术国家重点实验室培育基地, 杭州 310032)

\begin{abstract}
摘要：采用循环伏安法比较了玻碳、光亮银和粗粘化银电极对 $2,4,6$-三溴苯酚还原脱卤反应的电催化活性, 初 步研究了碱性水溶液中 2,4,6-三溴苯酚在粗䊁化银电极上的还原脱卤历程; 在此基础上, 利用恒电位电解法进一 步探索了 2,4,6-三溴苯酚在粗糙化银电极上的电还原脱卤历程. 结果表明, 粗糙化银电极对 2,4,6-三溴苯酚的选 择性还原脱卤反应具有优良的电催化活性, 且其活性优于光亮银和玻碳电极; $2,4,6$-三溴苯酚在粗鋉化银电极上 的还原反应是个逐步脱卤过程, 推测得出其邻位和对位 $\mathrm{C}-\mathrm{Br}$ 键的还原断裂所需的活化能非常接近, 而反应中 间产物 2,4-二溴苯酚的对位 $\mathrm{C}$ - $\mathrm{Br}$ 键的还原断裂所需的活化能比邻位要低; $2,4,6$-三溴苯酚能实现完全脱卤生 成苯酚, 主要路径为 $2,4,6$-三溴苯酚 $\rightarrow 2,4$-二溴苯酚 $\rightarrow 2$-溴苯酚 $\rightarrow$ 苯酚.
\end{abstract}

关键词：选择性脱卤； 2,4,6-三溴苯酚；银电极；废水处理

中图分类号: 0646

\section{Selective Dehalogenation of 2,4,6-Tribromophenol in Aqueous Solution on a Silver Cathode}

\author{
ZHU Ying-Hong XU Ying-Hua MA Hao ZHAO Feng-Ming MA Chun-An * \\ (State Key Laboratory Breeding Base of Green Chemistry-Synthesis Technology, College of Chemical Engineering and Materials, \\ Zhejiang University of Technology, Hangzhou 310032, P. R. China)
}

\begin{abstract}
The electrocatalytic activity of a roughened silver electrode towards the reductive dehalogenation of 2,4,6-tribromophenol was compared to a glassy carbon electrode and a polished silver electrode in basic aqueous solution by cyclic voltammetry (CV). Furthermore, the course of reductive dehalogention of 2,4,6-tribromophenol was studied by CV and controlled-potential electrolysis. Results indicated that roughened silver exhibited more powerful electrocatalytic activity than the glassy carbon electrode and the polished silver electrode for the selective dehalogention of 2,4,6-tribromophenol. The reduction of 2,4,6-tribromophenol was determined to be a stepwise dehalogenation as follows: 2,4,6-tribromophenol $\rightarrow$ 2,4-dibromophenol $\rightarrow$ 2-bromophenol $\rightarrow$ phenol. In addition, the activity energy needed for the dissociative reduction of the ortho carbon-halogen bond was similar to the para carbon-halogen bond for 2,4,6tribromophenol and a higher energy was probably required for 2,4-dibromophenol to cleave the ortho carbon-halogen bond compared to the para carbon-halogen bond.
\end{abstract}

Key Words : Selective dehalogenation; 2,4,6-tribromophenol; Silver electrode; Wastewater treatment

卤代芳烃及其衍生物化学性质稳定, 易在生物 体内累积, 具有较高的毒性和较强的致癌、致突变和 致畸作用, 相当一部分被列为美国 EPA(Environmental Protection Ageney)环境优先控制污染物, 一旦
进人环境将对人类及生态环境造成长期威胁, 因此 卤代芳烃的治理技术日益引起全球的关注 ${ }^{[1-10]}$. 卤代 芳烃通常以 Lewis 酸的形式存在难以失去电子, 而 且氧化不完全容易产生毒性更大、稳定性更强的产

Received: October 29, 2008; Revised: March 19, 2009; Published on Web: April 10, 2009.

*Corresponding author. Email: science@ zjut.edu.cn; Tel/Fax: +86-571-88320813.

国家基础研究重大项目前期研究专项基金(2003CCA01300)和浙江工业大学校级科学研究基金(X1018103)资助

C Editorial office of Acta Physico-Chimica Sinica 
物 ${ }^{[1,2]}$, 氧化处理常常难以奏效, 所以还原脱卤处理逐 渐受到重视. 还原脱卤的方法主要有生物厌氧还 原 ${ }^{[3,4]}$ 、零价态金属还原 ${ }^{[5-8]}$ 和电化学还原 ${ }^{[9-18]}$.

生物戻氧还原主要是通过篮选或构建高效菌 株, 通过添加供电子体(通常是易降解化合物)进行 还原. 该方法虽然廉价, 但是反应条件过于苛刻, 且 还原效率较低, 因此其适用范围非常有限.

零价态金属还原方法分单金属还原 ${ }^{[5}$ 和双金属 还原两种 ${ }^{[6-8]}$. 单金属还原虽然简单廉价, 但是还原 活性不高 (如 $\mathrm{Fe} / \mathrm{Fe}^{2+}$ 标准电势为 $-0.45 \mathrm{~V}$ ), 这使得卤 代芳烃还原需要在酸性甚至强酸性环境下进行, 且 对相当部分卤代芳烃不具有还原能力, 同时金属易 形成表面惰性层(氢氧化物)使活性不断降低, 并产 生大量金属泥等二次污染; 在贵金属的协同作用下 (催化加氢), 双金属还原活性得到极大的提高, 但贵 金属的回收成为了另一个难题.

与其他还原方法相比, 电化学还原反应条件更 加温和、反应选择性更高, 但是多数卤代芳烃的还原

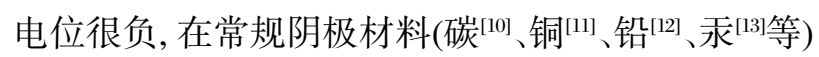
上很难实现还原. Pd 等贵金属电极对卤代芳烃的还 原具有较高的电催化活性, 但电流效率仍然较低 ${ }^{[14-17]}$. 最近的研究表明银电极对卤代芳烃的还原脱卤反应 具有很强的电催化活性 ${ }^{[18,19]}$, 银电极对卤代芳烃具有 特殊的吸附力, 两者形成的吸附态能使卤代芳烃还 原过程的活化能显著降低. 但奇怪的是, 很少有文献 报道水溶液中卤代芳烃在银电极上的电化学还原脱 卤反应 ${ }^{[20]}$.

本文选用 2,4,6-三溴苯酚作为卤代芳烃的模型 污染物, 研究碱性水溶液中 $2,4,6$-三溴苯酚在银电 极上的选择性还原脱卤反应, 初步得出了 $2,4,6$-三 溴苯酚的电化学还原脱卤历程, 希望能为地下水及 废水中含卤代芳烃的电催化还原脱卤技术提供理论 依据.

\section{1 实验部分}

\section{1 电化学测试}

循环伏安曲线采用恒电位仪(PAR Model 273 英国)测定. 电解池采用三电极体系, 阴、阳极室间用 多孔烧结玻璃分隔, 工作电极分别为粗鋉化银电极 $(\operatorname{Ag}(\mathrm{r}))(\phi=2 \mathrm{~mm})$ 、光亮银电极 $(\mathrm{Ag}(\mathrm{p}))(\phi=2 \mathrm{~mm})$ 和玻 碳电极 $(\phi=2 \mathrm{~mm})$, 辅助电极为大面积铂片, 参比电极 为饱和甘录电极 (SCE), 电解液为 $0.5 \mathrm{~mol} \cdot \mathrm{L}^{-1} \mathrm{NaOH}$ 水溶液, 实验温度 $(25 \pm 1){ }^{\circ} \mathrm{C}$. 文中的电极电位值均相
对于饱和甘录电极. 2,4,6-三溴苯酚, 2,4-二溴苯酚, 2,6-二溴苯酚, 2-溴苯酚和 4-溴苯酚等试剂由阿法埃 莎(天津)化学有限公司提供, 纯度均在 $98.0 \%$ 以上. 玻碳电极和光亮银电极的预处理方法及粗粘化银电 极的制备方法参见文献[20].

\section{2 恒电位电解}

采用恒电位仪(PAR Model 273 英国)进行恒电 位电解实验. 电解实验在两腔室电解池中进行, 电解 池的阴、阳极室用阳离子膜 $\left(\right.$ Nafion $\left.{ }^{\circledR} 117\right)$ 分隔, 阴、 阳极室的体积均为 $50 \mathrm{~mL}$. 阴极为粗糙化银电极 $(2$ $\mathrm{cm} \times 3 \mathrm{~cm} \times 0.1 \mathrm{~cm})$, 阳极为光亮铂电极 $(2 \mathrm{~cm} \times 3 \mathrm{~cm} \times$ $0.05 \mathrm{~cm}$ ), 参比电极为饱和甘录电极. 阴极液为 0.5 $\mathrm{mol} \cdot \mathrm{L}^{-1} \mathrm{NaOH}+10 \mathrm{mmol} \cdot \mathrm{L}^{-1}$ 溴代苯酚的水溶液 $(40$ $\mathrm{mL})$, 阳极液为 $0.5 \mathrm{~mol} \cdot \mathrm{L}^{-1} \mathrm{NaOH}$ 的水溶液 $(40 \mathrm{~mL})$. 电解过程中温度控制在 $(25 \pm 2){ }^{\circ} \mathrm{C}$, 阴极液用磁力摚 拌器进行激烈搅拌, 阳极液保存静止.

\section{3 产物分析}

产物分析分三步进行, (1) 取 $1 \mathrm{~mL}$ 电解液, 用浓 盐酸酸化到 $\mathrm{pH} \approx 1.0$, 然后用去离子水稀释到 $5 \mathrm{~mL}$; (2) 用 $10 \mathrm{~mL}$ 乙醚分两次对酸化电解液进行萃取, 然 后混合萃取剂; (3) 取萃取剂 $(25 \mu \mathrm{L})$ 进样. 电解产物 用配置有色谱柱 (Symmetry ${ }^{\circledR}$ Columns: $250 \mathrm{~mm}$ length $\times 4.6 \mathrm{~mm}$ i.d., $5 \mu \mathrm{m}$ particle size) 和光电二极管 检测器(2996 Photodiode Array Detector)的高效液相 色谱仪 (Waters) 测定. 流动相为三氟甲酸、甲醇、水, 其体积比为 1:500:500, 测定波长为 $285 \mathrm{~nm}$.

\section{2 结果与讨论}

\section{1 循环伏安曲线}

图 1 为 2,4,6-三溴苯酚在银电极和玻碳电极上 的循环伏安曲线. 由图可见, 在玻碳电极上除了析氢 电流外没有明显的电流响应, 说明 2,4,6-三溴苯酚 在玻碳电极上不容易实现还原. 相对玻碳电极, 银电 极表现出了良好的电催化活性. $\operatorname{Ag}(\mathrm{p})$ 电极上出现了 一个明显的还原峰 $\left(E_{\mathrm{p}}=-1.27 \mathrm{~V}\right)$ 而没有相对应的氧 化峰, 说明 2,4,6-三溴苯酚在 $\mathrm{Ag}(\mathrm{p})$ 电极上的还原反 应是不可逆的. 与 $\operatorname{Ag}(\mathrm{p})$ 电极不同, $\operatorname{Ag}(\mathrm{r})$ 电极不但具 有更大的背景电流密度 (双电层充电引起) 而且有两 个电位更正的还原峰 $\left(E_{\mathrm{p}}=-1.03,-1.17 \mathrm{~V}\right)$, 这表明 $\mathrm{Ag}(\mathrm{r})$ 电极的电催化活性高于 $\mathrm{Ag}(\mathrm{p})$ 电极.

大量研究表明 ${ }^{[18-20]}$, 多数卤代有机物在银电极 上的还原反应遵循过渡态机理. 在过渡态机理中, 最 为关键的步骤是有机物分子上卤原子和银电极的相 


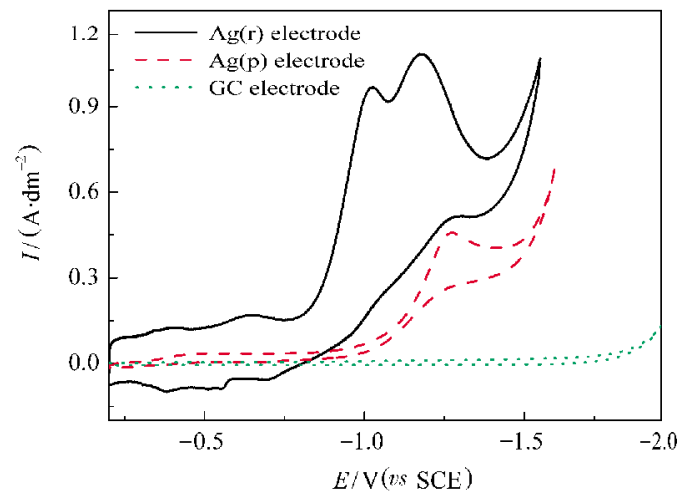

图 $12,4,6$-三溴苯酚的循环伏安曲线

Fig.1 Cyclic voltammograms of 2,4,6-tribromophenol temperature: $298 \mathrm{~K}$; electrolyte: $10 \mathrm{mmol} \cdot \mathrm{L}^{-1} 2,4,6$-tribromophenol+ $0.5 \mathrm{~mol} \cdot \mathrm{L}^{-1} \mathrm{NaOH}$ aqueous solution; scan rate: $100 \mathrm{mV} \cdot \mathrm{s}^{-1}$; "GC electrode" represents "glassy carbon electrode", " $\mathrm{Ag}(\mathrm{p})$ electrode" represents "polished silver electrode" and "Ag(r) electrode" represents "roughened silver electrode"

互作用步骤, 这种相互作用能促使卤代有机物吸附 到银电极上, 进而实现 $\mathrm{C}-\mathrm{X}$ 键的还原断裂, 正是因 为这种相互作用, 银电极对还原脱卤反应才具备了 优良的电催化性能. 粗糙化处理能进一步提高银电 极对 2,4,6-三溴苯酚还原反应的电催化活性说明电 极的表面形貌特征是决定相互作用大小的主要因素 之一.

图 2 为三种溴代苯酚 $(2,4,6$-三溴苯酚、2,4-二溴 苯酚、2-溴苯酚)在银电极上的循环伏安曲线. 与快 速电势扫描时不同(图 $1 \mathrm{a}, 100 \mathrm{mV} \cdot \mathrm{s}^{-1}$ ), 在较慢的扫 描速度下 $\left(25 \mathrm{mV} \cdot \mathrm{s}^{-1}\right), 2,4,6$-三溴苯酚还原过程中出

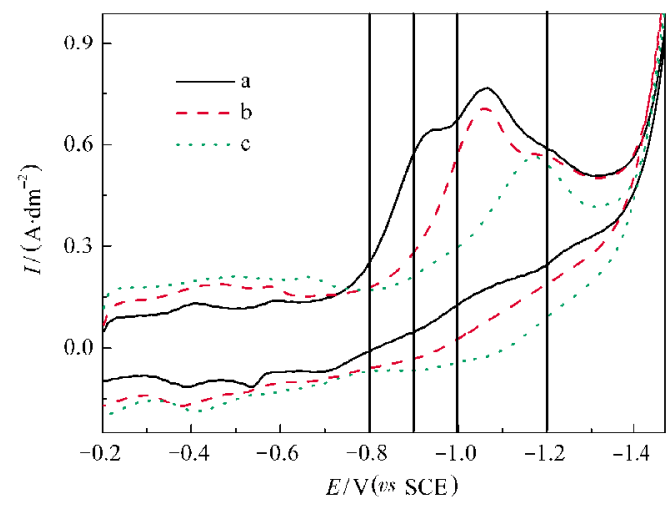

图 2 (a) 2,4,6-三溴苯酚、(b) 2,4-二溴苯酚、(c) 2-溴苯酚在 粗粘化银电极上的循环伏安曲线

Fig.2 Cyclic voltammograms of (a) 2,4,6tribromophenol, (b) 2,4-dibromophenol, (c) 2bromophenol on roughened silver electrode temperature: $298 \mathrm{~K}$; electrolyte: $10 \mathrm{mmol} \cdot \mathrm{L}^{-1}$ bromophenols + $0.5 \mathrm{~mol} \cdot \mathrm{L}^{-1} \mathrm{NaOH}$ aqueous solution; scan rate: $25 \mathrm{mV} \cdot \mathrm{s}^{-1}$
现了三个不可逆的还原峰 $\left(E_{\mathrm{p}}=-0.93,-1.08,-1.17 \mathrm{~V}\right)$, 这可能是因为在扫描速度较慢时电极表面会产生更 多的还原中间产物(如 2,4-二溴苯酚和 2-溴苯酚等), 新出现的还原峰 $\left(E_{\mathrm{p}}=-1.17 \mathrm{~V}\right)$ 可能对应于 2 -溴苯酚 的还原.

进一步比较三种底物的循环伏安曲线, 可以发 现三个显著的特征: (1) 溴代苯酚的还原峰数目和苯 环上溴原子的数目相同; (2) 苯环上的溴原子越多, 溴代苯酚的第一个还原峰电位越正; (3) 2-溴苯酚的 还原峰与 2,4-二溴苯酚和 2,4,6-三溴苯酚的最后一 个还原峰电位 $(-1.17 \mathrm{~V})$ 一致, $2,4-$ 二溴苯酚的第一个 还原峰和 2,4,6-三溴苯酚第二个还原峰电位 $(-1.08$ V)相一致. 这三个特征表明, (1) 溴代苯酚苯环上的 溴原子是逐个分步进行还原的; (2) 溴代苯酚苯环上 溴原子能增强溴代苯酚和银电极间的相互作用, 溴 原子越多则这种相互作用越强; (3) 2,4,6-三溴苯酚主 要的还原路径可能是: $2,4,6$-三溴苯酚 $\rightarrow 2,4$-二溴 苯酚 $\rightarrow 2$-溴苯酚 $\rightarrow$ 苯酚.

\section{2 恒电位电解实验}

为了进一步探索溴代苯酚在银电极上的还原脱 溴路径, 我们进行了 $2,4,6$-三溴苯酚的恒电位电解 实验. 图 3 和图 4 分别显示了 $2,4,6$-三溴苯酚理论 上可能的还原脱溴路径和 2,4,6-三溴苯酚在恒电位 电解过程中高效液相色谱(HPLC)结果.

如图3所示, 2,4,6-三溴苯酚彻底还原脱卤生成 苯酚有三种可能途径, 在这三种途径中可能产生的 中间产物有四种:2,4-二溴苯酚, 2,6-二溴苯酚, 2-溴 苯酚和4-溴苯酚. 由图4可见, 电解过程中, 2,4-二溴 苯酚和 2 -溴苯酚分别是最主要的二溴苯酚和一溴苯 酚, 这进一步确证 2,4,6-三溴苯酚的主要还原路径为 $2,4,6$-三溴苯酚 $\rightarrow 2,4$-二溴苯酚 $\rightarrow 2$-溴苯酚 $\rightarrow$ 苯酚.

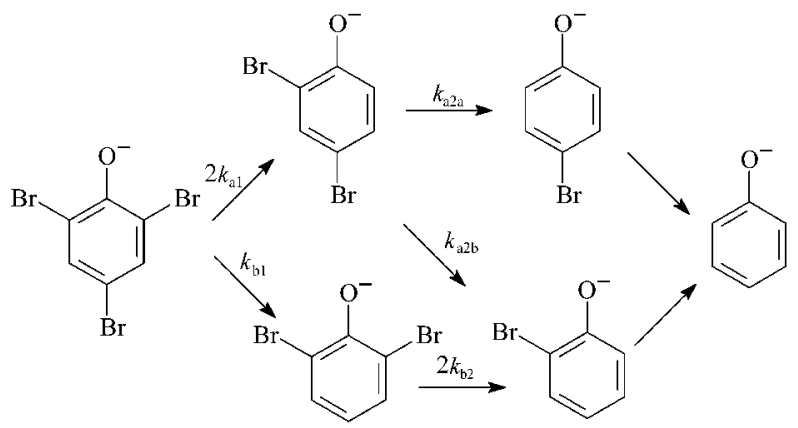

图 $32,4,6$-三溴苯酚在银电极上的还原脱溴路径

Fig.3 Dehalogenation pathways of 2,4,6tribromophenol in basic aqueous solution on silver cathode 


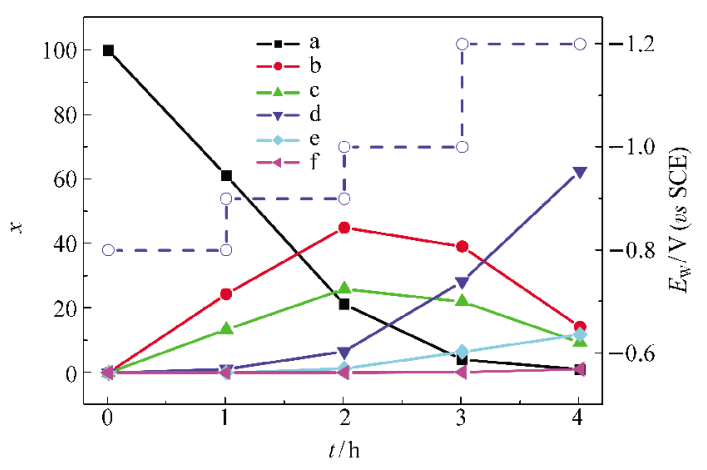

图 4 2,4,6-三溴苯酚在恒电位电解过程中高效液相色谱结果

Fig.4 HPLC monitoring of the reduction of 2,4,6tribromophenol $\left(10 \mathrm{mmol} \cdot \mathrm{L}^{-1}\right)$ on $\mathrm{Ag}(\mathrm{r})$ electrode in $0.5 \mathrm{~mol} \cdot \mathrm{L}^{-1} \mathrm{NaOH}$

(—, left coordinate) the molar fraction $x$ of (a) 2,4,6-tribromophenol, (b) 2,4-dibromophenol, (c) 2,6-dibromophenol, (d) 2-bromophenol, (e) 4-bromophenol, (f) phenol; (---, right coordinate) the working potential $E_{\mathrm{w}}$ as a function of the time of electrolysis

在第 $1 \mathrm{~h}$ 的电解过程中 (电极电位为 $-0.8 \mathrm{~V}$, 图 4), 生成的主要产物为 2,4-二溴苯酚 $(24.39 \%)$ 和 2,6-二 溴苯酚 $(13.38 \%)$, 说明 $2,4,6$-三溴苯酚的第一个还原 峰(图2a)对应于其邻位和对位单个溴的还原. 根据 2,4-二溴苯酚和 2,6-二溴苯酚的含量可以进一步得 出 $k_{\mathrm{al}}=0.91 k_{\mathrm{bl}}$, 说明 $2,4,6$-三溴苯酚邻位和对位 $\mathrm{C}$ $\mathrm{Br}$ 键的还原断裂所需的活化能非常接近. 在第 $2 \mathrm{~h}$ 的电解过程中 (电极电位为 $-0.9 \mathrm{~V}$ ), 2,4-二溴苯酚和 2,6-二溴苯酚的含量进一步增加并出现少量的 2-溴 苯酚 $(6.60 \%)$ 和 4-溴苯酚(1.26\%). 这说明 2,4,6-三溴 苯酚的第二个还原峰(图 2a) 可能对应于 2,4-二溴苯 酚的 4 位溴或 2,6-二溴苯酚的 6 位溴的还原. 在第 $3 \mathrm{~h}$ 的电解过程中 (电极电势为- $-1.0 \mathrm{~V}$ ), 2-溴苯酚和 4-溴苯酚的含量继续增加, 而 2,4-二溴苯酚和 2,6-二 溴苯酚的含量则开始减少, 说明此时二溴苯酚还原 为一溴苯酚的反应速率超过了三溴苯酚还原为二溴 苯酚的反应.

在第 $4 \mathrm{~h}$ 的电解过程中 (电极电势为 $-1.2 \mathrm{~V}$ ), 根 据 2,4-二溴苯酚, 2,6-二溴苯酚, 2-溴苯酚和 4-溴苯 酚四种中间产物的含量变化可以得到如下方程式

$$
\begin{aligned}
& \Delta C_{2}=6.29 \Delta C_{4} \\
& -\Delta C_{2,4}=-1.97 \Delta C_{2,6}
\end{aligned}
$$

上式中 $\Delta C_{2} 、 \Delta C_{4} 、 \Delta C_{2,4} 、 \Delta C_{2,6}$ 分别为 2 -溴、4-溴、2,4二溴和 2,6-二溴苯酚在第 $4 \mathrm{~h}$ 的电解过程中的增量. 根据恒电位电解的反应速率方程 ${ }^{[21]}$, 方程式(1)和(2) 可以转换为

$$
\begin{aligned}
& \int_{0}^{1}\left(k_{\mathrm{a} 2 \mathrm{~b}} \times C_{2,4}+2 k_{\mathrm{b} 2} \times C_{2,6}\right) \mathrm{d} t=6.29 \times \int_{0}^{1} k_{\mathrm{a} 2 \mathrm{a}} \times C_{2,4} \mathrm{~d} t \\
& \int_{0}^{1}\left(k_{\mathrm{a} 2 \mathrm{a}}+k_{\mathrm{a} 2 \mathrm{~b}}\right) \times C_{2,4} \mathrm{~d} t=1.97 \times \int_{0}^{1} 2 k_{\mathrm{b} 2} \times C_{2,6} \mathrm{~d} t
\end{aligned}
$$

上式中 $k_{\mathrm{a} 1 、} k_{\mathrm{b} 1} 、 k_{\mathrm{a} 2 a} 、 k_{\mathrm{a} 2 \mathrm{~b}} 、 k_{\mathrm{b} 2}$ 分别为 $2,4,6$-三溴苯酚还 原为 2,4-二溴苯酚, 2,4,6-三溴苯酚还原为 2,6-二溴 苯酚, 2,4-二溴苯酚还原为 4-溴苯酚, 2,4-二溴苯酚 还原为 2 -溴苯酚和 2,6 -二溴苯酚还原为 2 -溴苯酚的 反应速率常数. 对方程式(3)和(4)进行求解可得

$k_{\mathrm{a} 2 \mathrm{~b}}=3.84 k_{\mathrm{a} 2 \mathrm{a}}$

方程式(5)表明, 2,4-二溴苯酚对位 C一 $\mathrm{Br}$ 键还原断 裂所需的活化能比邻位低, 这和 Rondinini 等人 ${ }^{[2]}$ 的 实验结果 (2,4-二溴苯酚邻位溴比对位更容易还原) 不同, 这可能是由于两个体系的 $\mathrm{pH}$ 值不同. 根据卤 代有机物在银电极上还原的过渡态机理, 2,4-二溴 苯酚只有通过相互作用吸附到电极上形成特殊的吸 附态 $(\mathrm{R} \cdots \mathrm{Br} \cdots \mathrm{Ag})$ 后才能得到电子实现还原. 在中 性溶剂(乙腈+四乙基高氯酸铵)中, 2,4-二溴苯酚主 要以分子形态存在, 由于推电子共轭效应, 分子态 2,4-二溴苯酚的差基氢带部分正电, 它能和带负电 的银电极发生吸附作用从而辅助 2,4-二溴苯酚和银 电极产生吸附中间态 $(\mathrm{R} \cdots \mathrm{Br} \cdots \mathrm{Ag})^{[2,19]}$, 因为邻位 溴更靠近羟基氢，所以优先形成的吸附中间态 $(\mathrm{R} \cdots$ $\mathrm{Br} \cdots \mathrm{Ag}$ )发生在邻位溴和银电极之间, 因此邻位 $\mathrm{C}-$ $\mathrm{Br}$ 键更容易发生还原断裂; 在碱性水溶液中 $($ 水+氢 氧化钠), 2,4-二溴苯酚则主要以阴离子形式存在, 由 羟基氢的离去而形成的氧负离子 $\left(\mathrm{O}^{-}\right)$, 具有三对孤 对电子, 其带很强的负电, 与银电极之间有很强的 静电排斥作用, 这使更靠近氧负离子 $\left(\mathrm{O}^{-}\right)$的邻位溴 不容易接近银电极从而抑制吸附中间态 $(\mathrm{R} \cdots \mathrm{Br} \cdots$ $\mathrm{Ag}$ ）的形成, 因此邻位 $\mathrm{C}-\mathrm{Br}$ 键更难发生还原断裂. 另外, 与中性环境的差基相比, 碱性环境的氧负离子 $\left(\mathrm{O}^{-}\right)$带更多负电, 因此其具有更大的推电子能力, 这 使碱性环境的邻位溴通过吸电子诱导效应获得了更 多的电子, 因此邻位 $\mathrm{C}-\mathrm{Br}$ 键具有更高的电子云密 度, 其更不容易发生还原断裂.

\section{3 结 论}

循环伏安实验和恒电位电解实验表明, 粗糙化 银电极对 2,4,6-三溴苯酚在碱性水溶液中的还原脱 
溴反应具有优良的电催化性能, 电极表面形貌特征 是决定银电极电催化活性高低的一个主要因素, $2,4,6$-三溴苯酚的溴原子能逐个在银电极上还原最 后完全脱溴生成苯酚. 根据现有的实验数据我们推 测, 2,4,6-三溴苯酚的主要还原路径为 2,4,6-三溴苯 酚 $\rightarrow 2,4-$ 二溴苯酚 $\rightarrow 2$-溴苯酚 $\rightarrow$ 苯酚, 2,4,6-三溴苯 酚邻位和对位 $\mathrm{C}-\mathrm{Br}$ 键的还原断裂所需的活化能 非常接近, 而中间产物 2,4-二溴苯酚的对位 $\mathrm{C}-\mathrm{Br}$ 键还原断裂所需的活化能比邻位低.

\section{References}

1 Barbeau, C.; Deschenes, L.; Karamanev, D.; Comeau, Y.; Samson, R. Appl. Microbiol. Biotechnol., 1997, 48: 745

2 Rondinini, S.; Mussini, P. R.; Specchia, M.; Vertova, A. J. Eletrochem. Soc., 2001, 148(7): D102

3 Booker, R. S.; Pavlostathis, S. G. Water Res., 2000, 34(18): 4437

4 Kao, C. M.; Chai, C. T.; Liu, J. K.; Yeh, T. Y.; Chen, K. F.; Chen, S. C. Water Res., 2004, 38(3): 663

5 Matheson, L. J.; Tratnyek, P. G. Environ. Sic. Technol., 1994, 28 (12): 2045

6 Zhou, H. Y.; Xu, X. H. Journa of Chemical Industry and Engineering (China), 2004, 55(11): 1912 [周红艺, 徐新华. 化工学报, 2004, 55(11): 1912]

7 Patel, U. D.; Suresh, S. J. Hazard. Mater., 2007, 147(2): 431

8 Tong, S. P.; Hu, L. H.; Wei, H.; Ma, C. A. Environmental Science, 2005, 26(4): 59 [童少平, 胡丽华, 魏 红, 马淳安. 环境科学, 2005, 26(4): 59]

9 Sun, Z. R.; Li, B. H.; Hu, X.; Shi, M.; Ge, H.; Peng, Y. Z. Journal of Chemical Industry and Engineering (China), 2008, 59(5): 1271
[孙治荣, 李保华, 胡 翔, 石 敏, 葛 惠, 彭永臻. 化工学报, 2008, 59(5): 1271]

10 Kulikov, S. M.; Plekhanov, V. P.; Tsyganok, A. I.; Schlimm, C.; Heitz, E. Electrochimica Acta, 1996, 41(4): 527

$11 \mathrm{Xu}$, W. Y.; Gao, T. Y.; Zhou, R. F.; Ma, L. M. Environmental Science, 2005, 26(4): 51 [徐文英, 高廷耀, 周荣丰, 马鲁铭. 环境科学, 2005, 26 (4): 51]

12 Ross, N. C.; Spackman, R. A.; Hitchman, M. L.; White, P. C. J. Appl. Electrochem., 1997, 27: 51

13 Merica, S. G.; Bunce, N. J. Jedral, W.; Lipkowski, J. J. Appl. Electrochem., 1998, 28(6): 645

14 Cui, C. Y.; Quan, X.; Chen, S.; Zhao, H. M.; Li, X. Y.; Cui, X. Y.; Zhao, Y. Z. Journal of Safety and Environment, 2005, 5(4): 51 [崔春月, 全 姟, 陈 硕, 赵惠敏, 李新勇, 崔昕毅, 赵雅芝. 安全与环境学报, 2005, 5(4): 51]

15 Tsyganok, A. I.; Otsuka, K. Appl. Catal. B, 1999, 22(1): 15

16 Yang, B.; Yu, G.; Huang, J. Environ. Sci. Technol., 2007, 41: 7503

17 Yang, B.; Yu, G.; Huang J. Acta Phys. -Chim. Sin., 2006, 22(3): 306 [杨 波, 余 刚, 黄 俊. 物理化学学报, 2006, 22(3): 306]

18 Bellomunno, C.; Bonanomi, D.; Falciola, L.; Longhi, M.; Mussini, P. R.; Doubova, L. M.; Silvestro, G. D. Electrochimica Acta, 2005, 50(11): 2331

19 Rondinini, S.; Mussini, P. R.; Muttini, P.; Sello, G. Electrochimica Acta, 2001, 46(21): 3245

20 Xu, Y. H.; Zhu, Y. H.; Zhao, F. M.; Ma, C. A. Appl. Catal. A, 2007, 324: 83

21 Bard, A. J.; Faulkner, L. R.; Electrochemical methods: fundamentals and applications. 2th ed. New York: John Wiley \& Sons, Inc., 2001: 423-425 